AperTO - Archivio Istituzionale Open Access dell'Università di Torino

\title{
Multiple ADH genes are associated with upper aerodigestive cancers
}

\section{This is the author's manuscript}

Original Citation:

Availability:

This version is available http://hdl.handle.net/2318/69454

since

Published version:

DOI:10.1038/ng.151

Terms of use:

Open Access

Anyone can freely access the full text of works made available as "Open Access". Works made available under a Creative Commons license can be used according to the terms and conditions of said license. Use of all other works requires consent of the right holder (author or publisher) if not exempted from copyright protection by the applicable law. 


\section{\& Multiple $A D H$ genes are associated with upper aerodigestive cancers}

\author{
Mia Hashibe ${ }^{1}$, James D McKay ${ }^{1}$, Maria Paula Curado ${ }^{1,2}$, \\ Jose Carlos Oliveira ${ }^{2}$, Sergio Koifman ${ }^{3}$, Rosalina Koifman ${ }^{3}$, \\ David Zaridze $^{4}$, Oxana Shangina ${ }^{4}$, Victor Wünsch-Filho ${ }^{5}$, \\ Jose Eluf-Neto ${ }^{5}$, Jose Eduardo Levi ${ }^{5}$, Elena Matos ${ }^{6}$, \\ Pagona Lagiou ${ }^{7}$, Areti Lagiou ${ }^{7}$, Simone Benhamou ${ }^{8}$, \\ Christine Bouchardy ${ }^{9}$, Neonilia Szeszenia-Dabrowska ${ }^{10}$, \\ Ana Menezes ${ }^{11}$, Marinel Mór Dall'Agnol ${ }^{11}$, Franco Merletti ${ }^{12}$, \\ Lorenzo Richiardi $^{12}$, Leticia Fernandez ${ }^{13}$, Juan Lence ${ }^{13}$, \\ Renato Talamini ${ }^{14}$, Luigi Barzan ${ }^{15}$, Dana Mates ${ }^{16}$, \\ Ioan Nicolae Mates $^{17}$, Kristina Kjaerheim ${ }^{18}$, Gary J Macfarlane ${ }^{19}$, \\ Tatiana V Macfarlane ${ }^{19}$, Lorenzo Simonato ${ }^{20}$, Cristina Canova ${ }^{20}$, \\ Ivana Holcátová ${ }^{21}$, Antonio Agudo ${ }^{22}$, Xavier Castellsagué ${ }^{22}$, \\ Ray Lowry ${ }^{23}$, Vladimir Janout ${ }^{24}$, Helena Kollarova ${ }^{24}$, \\ David I Conway ${ }^{25}$, Patricia A McKinney ${ }^{26,27}$, Ariana Znaor ${ }^{28}$, \\ Eleonora Fabianova $^{29}$, Vladimir Bencko ${ }^{21}$, Jolanta Lissowska ${ }^{30}$, \\ Amelie Chabrier ${ }^{1}$, Rayjean J Hung ${ }^{1,31}$, Valerie Gaborieau ${ }^{1}$, \\ Paolo Boffetta ${ }^{1}$ \& Paul Brennan ${ }^{1}$
}

\section{Alcohol is an important risk factor for upper aerodigestive cancers and is principally metabolized by alcohol dehydrogenase (ADH) enzymes. We have investigated six $A D H$ genetic variants in over 3,800 aerodigestive cancer cases and 5,200 controls from three individual studies. Gene variants rs1229984 $(A D H 1 B)$ and rs1573496 (ADH7) were significantly protective against aerodigestive cancer in each individual study and overall $\left(P=10^{-10}\right.$ and $10^{-9}$, respectively). These effects became more apparent with increasing alcohol consumption ( $P$ for trend $=0.0002$ and 0.065 , respectively). Both gene effects were independent of each other, implying that multiple $A D H$ genes may be involved in upper aerodigestive cancer etiology.}

The alcohol dehydrogenase $(\mathrm{ADH})$ pathway includes seven distinct $A D H$ genes, a key candidate gene group for aerodigestive cancers ${ }^{1-3}$.
Studies of aerodigestive cancer in populations of European origin have focused on $A D H 1 C$ with little evidence of any effect ${ }^{4}$. We previously reported an association for $A D H 1 B \mathrm{R} 48 \mathrm{H}$ (rs1229984) in a central European (CE) population ${ }^{5}$ and now consider the effect of this and five other $A D H$ variants in an expanded study comprising 809 aerodigestive cancer cases and 2,586 controls from the CE study as well as a further 3,067 aerodigestive cancer cases and 2,692 controls from two other studies in Europe (ARCAGE study) and Latin America (LA study) (total of 3,876 cases and 5,278 controls). All three studies were coordinated by the International Agency for Research on Cancer (IARC) and followed a similar protocol (Supplementary Methods online). Of the 3,876 cases, 1,790 were cancers of the oral cavity or pharynx, 1,659 were cancers of the hypopharynx or larynx and 427 were cancers of the esophagus (Supplementary Table 1 online). Cases with a histology other than squamous cell were excluded.

The HapMap Consortium has genotyped 163 SNPs in the vicinity of the $A D H$ gene cluster with a minor allele frequency (MAF) of $4 \%$ or more in the CEPH Utah (CEU) population ${ }^{6}$. Inspection of the linkage disequilibrium (LD) pattern across this region indicates that $A D H 1 A$, $A D H 1 B, A D H 1 C, A D H 4, A D H 5$ and $A D H 6$ are relatively highly correlated, whereas $A D H 7$ showed little correlation with the remaining six (Supplementary Fig. 1a online). From all verified missense SNPs in the seven $A D H$ genes found in both the NCBI SNP and SNP500 databases $^{7}$, we selected eight that had a MAF $>4 \%$ in the CEU population. Three missense SNPs in ADH4 (rs1126671, rs1126673 and rs1042364) were in strong LD, and thus were genotyped by the highly correlated tagging SNP rs1984362 $\left(r^{2}>0.89\right)$. In total, we genotyped six genetic variants (five missense SNPs and one tagging SNP) in all three studies (Table 1 and Supplementary Table 2 online).

In the pooled analysis on all 3,876 cases and 5,278 controls, four variants reported a significant association (Supplementary Table 3 online). The most prominent was with rs1229984 (in $A D H 1 B$; OR for codominant model $=0.59(95 \% \mathrm{CI}=0.50-0.69)$; $P$ under codominant model $\left.=8 \times 10^{-10}\right)$. This variant was significant in each of the three individual studies (CE: $P=5 \times 10^{-5}$; ARCAGE: $P=1 \times 10^{-4}$; LA: $P=0.002)$. A second strongly significant finding in the pooled analysis was with $\mathrm{rs} 1573496$ (in $A D H 7 ; \mathrm{OR}=0.69(0.61-0.78) ; P=$ $3 \times 10^{-9}$ ), a new potential susceptibility gene for this cancer. This variant was also significant in each of the three individual studies

\footnotetext{
${ }^{1}$ International Agency for Research on Cancer, Lyon, France. ${ }^{2}$ Hospital Araujo Jorge, Goiania, and Populational Cancer Register of Goiania, Brazil. ${ }^{3}$ Escola Nacional de Suade Publica, Rio de Janeiro, Brazil. ${ }^{4}$ Cancer Research Centre, Moscow, Russia. ${ }^{5}$ Universidade de Sao Paulo, Sao Paulo, Brazil. ${ }^{6}$ Institute of Oncology Angel H. Roffo, University of Buenos Aires, Argentina. ${ }^{7}$ University of Athens School of Medicine, Athens, Greece. ${ }^{8}$ Institut National de la Santé et de la Recherche Médicale U794, Evry, France. ${ }^{9}$ Geneva Cancer Registry, Geneva, Switzerland. ${ }^{10}$ Institute of Occupational Medicine, Lodz, Poland. ${ }^{11}$ Universidade Federal de Pelotas, Pelotas, Brazil. ${ }^{12}$ Unit of Cancer Epidemiology, CeRMS and University of Turin, Turin, Italy. ${ }^{13}$ Institute of Oncology and Radiobiology, Havana, Cuba. ${ }^{14}$ Aviano Cancer Centre, Aviano, Italy. ${ }^{15}$ General Hospital of Pordenone, Pordenone, Italy. ${ }^{16}$ Institute of Public Health, Bucharest, Romania. ${ }^{17}$ University of Medicine and Pharmacy "Carol Davila", Bucharest, Romania. ${ }^{18}$ Cancer Registry of Norway, Oslo, Norway. ${ }^{19}$ University of Aberdeen School of Medicine, Aberdeen, UK. ${ }^{20}$ University of Padua, Padova, Italy. ${ }^{21}$ Institute of Hygiene and Epidemiology, Prague, Czech Republic. ${ }^{22}$ Institut Català d'Oncologia, Barcelona, Spain. ${ }^{23}$ University of Newcastle Dental School, Newcastle, UK. ${ }^{24}$ Palacky University, Olomouc, Czech Republic. ${ }^{25}$ University of Glasgow Dental School, Glasgow, Scotland. ${ }^{26}$ University of Leeds Centre for Epidemiology and Biostatistics, Leeds, UK. ${ }^{27}$ NHS National Services Scotland, Information Services Division, Edinburgh, Scotland. ${ }^{28}$ Croatian National Cancer Registry, Zagreb, Croatia. ${ }^{29}$ Specialized State Health Institute, Banská Bystrica, Slovakia. ${ }^{30}$ The Maria Sklodowska-Curie Memorial Cancer Center and Institute of Oncology, Warsaw, Poland. ${ }^{31}$ School of Public Health, University of California at Berkeley, Berkeley, California 94720, USA. Correspondence should be addressed to P.B. (Brennan@iarc.fr).
} 
Table 1 Minor allele frequency (\%) of six $A D H$ candidate variants within each recruitment center

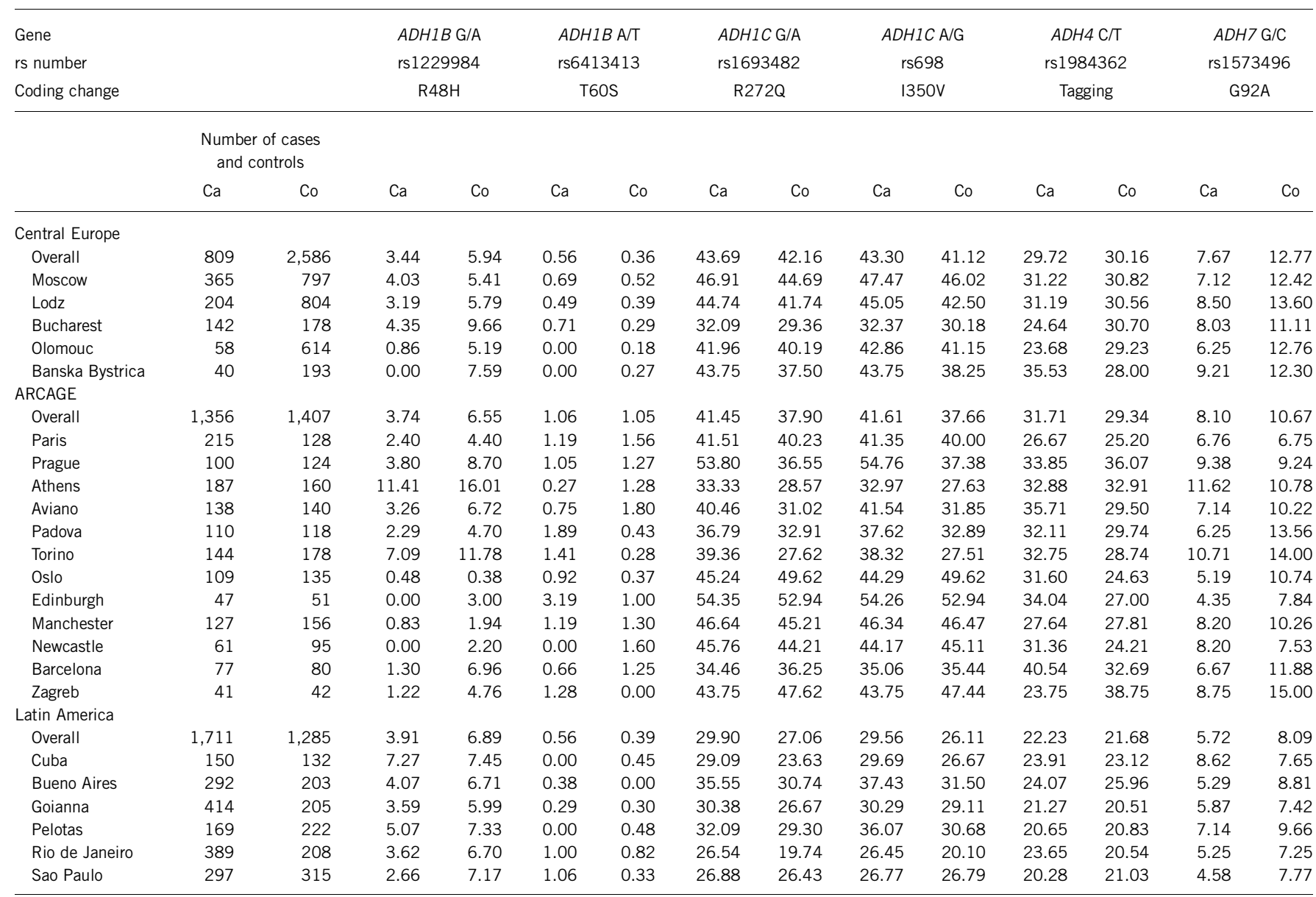

Ca, cases; Co, controls.

(CE: $P=1 \times 10^{-7}$; ARCAGE: $P=0.015$; LA: $\left.P=0.008\right)$. Significant effects were also observed for both $A D H 1 C$ variants: rs 1693482 $\left(\mathrm{OR}=1.17(1.09-1.26) ; P=2 \times 10^{-5}\right)$ and $\mathrm{rs698}(\mathrm{OR}=1.14$ (1.06-1.23); $\left.P=3 \times 10^{-4}\right)$. These two variants were highly correlated in all three studies $\left(D^{\prime}>0.99, r^{2}>0.97\right.$, Supplementary Fig. 1b), so we considered only rs1693482 for further analysis. A moderate increase in risk for this variant was observed in each of the three individual studies (CE: $P=0.02$; ARCAGE: $P=0.002$; LA: $P=0.04$ ).

Both rs1229984 (ADH1B) and $\operatorname{rs} 1693482(A D H 1 C)$ were in LD, with $D^{\prime}>0.75$ in all three studies (Supplementary Fig. 1b). In order to determine the independence of each SNP, we repeated analysis of rs1693482 (ADH1C) excluding rs1229984[A] (ADH1B) carriers and vice versa (Supplementary Table 4a online). This indicated that the effect of rs1229984 (ADH1B) was not influenced by rs1693482 $(A D H 1 C)$ and that the effect observed with rs1693482 (ADH1C) could not be explained solely by LD with rs1229984 (ADH1B). In contrast, there was little LD between rs1573496 (ADH7) and rs1229984 $(A D H 1 B)$, with $D^{\prime}<0.21$ in all three studies (Supplementary Fig. 1b). To illustrate their independence, we repeated analysis of rs1229984 (ADH1B) excluding carriers of rs1573496[C] $(A D H 7)$ and vice versa. As expected considering the lack of $\mathrm{LD}$ between these two SNPs, the risk estimates remained very similar to those based on the complete dataset (Supplementary Table $4 b$ ).

Subsequently, we focused on the effects of rs1229984[A] (ADH1B) and rs1573496[C] (ADH7) after stratifying by site of cancer, age, alcohol consumption, tobacco smoking and study (Fig. 1). Significant heterogeneity $(P=0.001)$ for $\operatorname{rs} 1229984(A D H 1 B)$ was observed by cancer site with a reduction in risk of between two- and threefold for oral and pharyngeal cancer $(\mathrm{OR}=0.45(0.35-0.57))$ and esophageal cancer $(\mathrm{OR}=0.34(0.20-0.56))$, as opposed to a $30 \%$ decrease in risk for larynx cancer $(\mathrm{OR}=0.71(0.57-0.88))$. We observed significant heterogeneity by site for $\operatorname{rs} 1573496$ ( $A D H 7)(P=0.023)$, with the most pronounced protective effect being for esophageal cancer $(\mathrm{OR}=0.45(0.32-0.64))$. For both gene variants, there was an increasing protective effect with increasing alcohol consumption. For rs1229984 $(A D H 1 B)$, we did not observe any effect among never drinkers $(\mathrm{OR}=1.02(0.66-1.56))$, whereas we observed an over twofold effect among those who drank above the median level of alcohol in each study $(\mathrm{OR}=0.42(0.31-0.56) ; P$ for trend $=0.0002)$. Similarly, for rs1573496 ( $A D H 7$ ), no effect was detected among never drinkers and a $40 \%$ decrease in risk was observed for heavy drinkers $(\mathrm{OR}=0.61(0.50-0.75) ; P$ for trend $=0.065)$. The effect of rs1229984 $(A D H 1 B)$ was consistent across the three studies, whereas the effect of rs1573496 $(A D H 7)$ varied from $\mathrm{OR}=0.54(0.43-0.69)$ in the $\mathrm{CE}$ study to $0.79(0.64-0.98)$ for the ARCAGE study ( $P$ for heterogeneity $=0.08$ ). Any potential heterogeneity seemed to be explained by a higher proportion of esophageal cancer cases in the CE study $(21 \%)$ as opposed to the LA study (9\%) and the ARCAGE study (8\%). When esophageal cancer cases were excluded from this analysis, no betweenstudy heterogeneity was apparent for $\operatorname{rs} 1573496(A D H 7)$ ( $P$ for heterogeneity $=0.38$ ). 

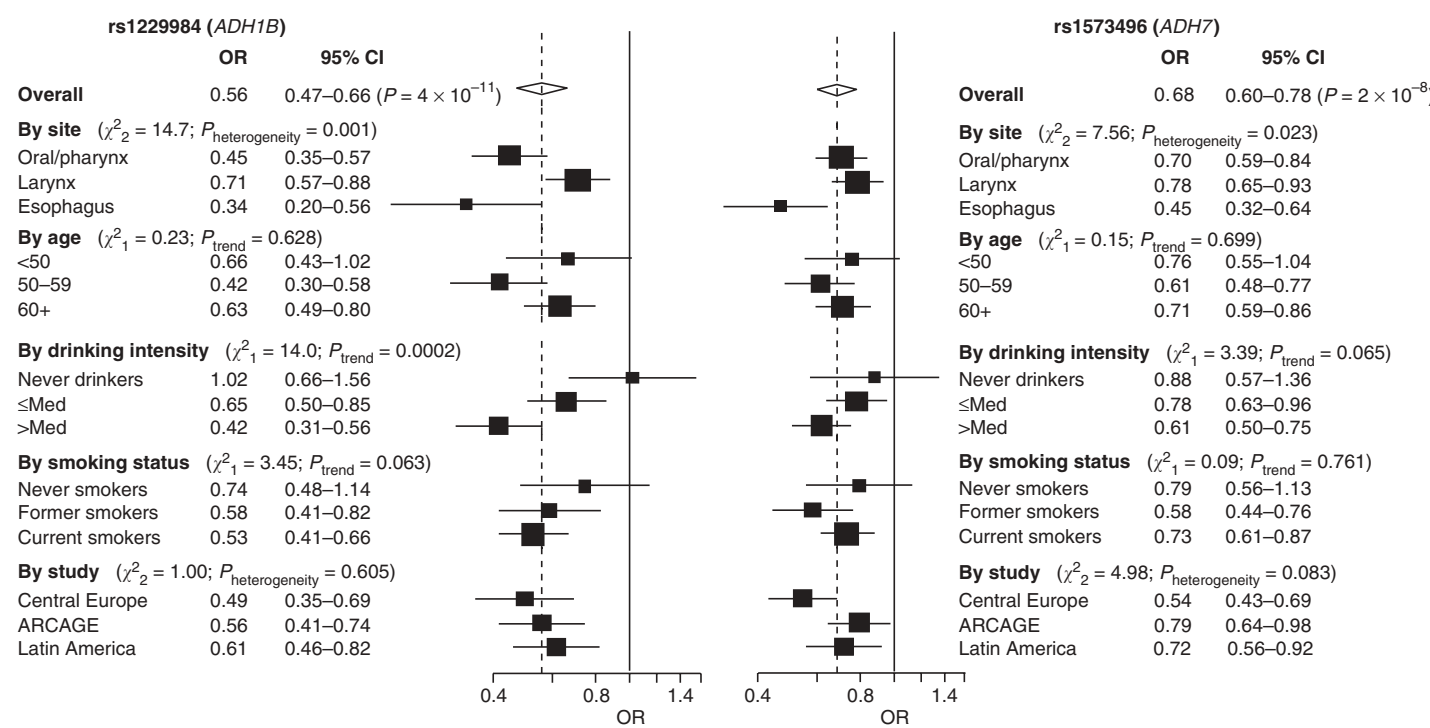

Figure 1 Odds ratio (OR) of upper aerodigestive cancer by rs1229984 (ADH1B) and rs1573496 (ADH7) genotypes. Rare allele carriers (dominant model) versus common allele homozygous genotype. ORs are standardized by age, sex, center, cumulative alcohol consumption and, when relevant, smoking. ORs and $95 \% \mathrm{Cl}$ are derived from fixed effects models.

We also analyzed the effect of carrying the rs1693482 (ADH1C) G/A or A/A variant after stratifying by site of cancer, age, alcohol consumption, tobacco smoking and study (Supplementary Fig. 2 online). No notable heterogeneity by any of these factors was observed.

Finally, we assessed the combined effect of carrying either one or both of the rare gene variants from $\operatorname{rs} 1229984(A D H 1 B)$ and rs1573496 (ADH7). For those who possessed only rs1229984[A] $(A D H 1 B)$, the OR for aerodigestive cancer was $0.56(0.45-0.70)$, and for those who possessed only rs1573496[C] (ADH7), the OR was 0.70 (0.61-0.82), whereas for those who possessed both rare gene variants, the OR was $0.45(0.34-0.60$; $P$ for trend in possessing zero, one or two variants $\left.=10^{-16}\right)$.

These results provide strong evidence that both $A D H 1 B$ and $A D H 7$ have an important association with susceptibility to aerodigestive cancer. The strong similarity of the results from different studies argues against population stratification or other biases. These effects seem to be relevant for all aerodigestive tract subsites, although they may be more prominent for esophageal cancer, and they both seem to be dependent on alcohol consumption - that is, among nondrinkers, the gene variants have little or no effect on disease risk, whereas among alcohol drinkers, the protective effect is more apparent at higher alcohol intake. Furthermore, neither gene variant seemed to be consistently associated with the amount of alcohol consumed in controls (data not shown), indicating that any protective effect from these gene-environment interactions is likely to be due to their role in changing the carcinogenic effect of alcohol beverages.

Whether we have studied the causal variants in these two genes or whether our associations are secondary to other causal variants is unknown. rs1229984 (ADH1B) G/A heterozygotes and A/A homozygotes are known to metabolize ethanol up to 100 times quicker than the common rs1229984 (ADH1B) G/G homozygote ${ }^{4}$, providing support that quick eradication of ethanol, and therefore lower local exposure, may be protective ${ }^{5}$. The potential role of $A D H 7$ is unclear, however, although variants in this gene may also influence ethanol metabolism ${ }^{8}$. Given the limited association between $A D H 7$ and other $A D H$ genes (Supplementary Fig. 1a), it would be expected that the causal association for this variant resides in the region of $A D H 7$.
In summary, our analysis of six $A D H$ genetic variants in over 3,800 cases and 5,000 controls has identified two that are independently and strongly associated with aerodigestive cancers. Elucidation of these findings and further detailed characterization of this pathway in a large series of subjects seems warranted.

Note: Supplementary information is available on the Nature Genetics website.

\section{ACKNOWLEDGMENTS}

First and foremost we thank all participants of the CE, LA and ARCAGE studies and the staff in the collaborating centers. Subject recruitment was supported by four grants from the European Commission (IC15-CT96-0313, IC15-CT98-0322, IC18-CT97-0222 and QLK1-CT2001-00182) and one grant from the World Cancer Research Fund (WCRF 99A28). Genotyping and analysis was funded by a National Cancer Institute R01 grant (contract no. CA 092039-01A2). The funding agencies did not have any involvement in the study design, collection, analysis and interpretation of the data or in the writing of report and decision to submit the paper.

\section{AUTHOR CONTRIBUTIONS}

P. Boffetta, P. Brennan, M.P.C., J.C.O., S.K., R.K., V.W.-F., J.E.N., E.M., A.M., M.M.D., L.F. and J. Lence jointly designed the LA study and organized the recruitment of participants. P. Brennan, P. Boffetta, D.Z., O.S., N.S.-D., D.M., I.N.M., I.H., V.J., H.K., E.F. and J. Lissowska jointly designed the CE study and organized the recruitment of participants. P. Brennan, M.H., P.L., A.L., S.B., C.B., F.M., L.R., R.T., L.B., K.K., G.J.M., T.V.M., L.S., C.C., A.A., X.C., R.L., D.I.C., P.A.M., A.Z. and V.B. jointly designed the ARCAGE study and organized the recruitment of participants. J.D.M., M.H., A.C., R.J.H., V.G., J.E.L. and P. Brennan organized biological sample storage. J.D.M., M.H., V.G. and P. Brennan conducted SNP selection and the statistical analysis. M.H., J.D.M. and P. Brennan drafted the manuscript, and all coauthors contributed to the final draft.

Published online at http://www.nature.com/naturegenetics/ Reprints and permissions information is available online at http://npg.nature.com/ reprintsandpermissions/

1. Carr, L.G. et al. Alcohol. Clin. Exp. Res. 13, 594-596 (1989).

2. Hoog, J.O. et al. Eur. J. Biochem. 159, 215-218 (1986).

3. Smith, M. Adv. Hum. Genet. 15, 249-290 (1986).

4. Brennan, P. et al. Am. J. Epidemiol. 159, 1-16 (2004).

5. Hashibe, M. et al. Cancer Epidemiol. Biomarkers Prev. 15, 696-703 (2006).

6. The International HapMap Project. Nature 426, 789-796 (2003).

7. Smigielski et al. Nucleic Acids Res. 28, 352-255 (2000).

8. Birley, A.J. et al. Hum. Mol. Genet. 17, 179-189 (2008). 